\title{
Chip-based quantum key distribution
}

\author{
Leong-Chuan Kwek ${ }^{1,2,3,4^{*}}$, Lin Cao ${ }^{4,5}$, Wei Luo ${ }^{4,6}$, Yunxiang Wang ${ }^{7}$, Shihai Sun ${ }^{8}$, Xiangbin Wang9,10,11 \\ and Ai Qun Liu 4,6
}

\begin{abstract}
Quantum key distribution is a matured quantum science and technology. Over the last 20 years, there has been substantial research and development in this area. Recently, silicon technology has offered tremendous promise in the field for improved miniaturization of quantum key distribution through integrated photonic chips. We expect further progress in this area both in terms of protocols, photon sources, and photon detectors. This review captures some of the recent advances in this area.
\end{abstract}

Keywords: Chip-based QKD, MDI, Quantum communications

\section{Introduction}

The need for secure transmission of information depends crucially on the level of paranoia in an organization. Thus, there has been a need to maintain secure transmission of information within government organizations, especially in defense, or large corporations, especially with regards to trade secrets. Yet, how do we transmit messages securely?

Cryptography, the ancient art of secret writing, has always been a combination of scrambling and confusion. A good scrambling of the plaintexts (messages) is the basis of many symmetric-key encryption schemes, like the Advanced Encryption Standard (AES). There is also asymmetric-key encryption algorithms or public key distribution systems that use a pair of keys, a public key associated with the creator or sender for encrypting messages and a private key that only the receiver (often the originator) knows for decrypting that information. One such public key scheme is the Rivest-Shamir-Adleman (RSA) protocol which relies essentially on the difficulty of factoring two large prime numbers.

Conceived much earlier (at least 10 years earlier), but finally published in 1983, Wiesner outlines a protocol for

\footnotetext{
*Correspondence: cqtklc@nus.edu.sg

${ }^{1}$ Centre for Quantum Technologies, National University of Singapore,

Singapore, Singapore

${ }^{2}$ MajuLab, CNRS-UNS-NUS-NTU International Joint Research Unit, Singapore

UMI 3654, Singapore, Singapore

Full list of author information is available at the end of the article
}

storing quantum money in two conjugate bases:the first one in vertical and horizontal polarization and the second one in right and left circular polarization [1]. Wiesner communicated this idea to Charles Bennett who subsequently proposed a quantum key distribution with Gille Brassard based on the idea of "conjugate" bases [2, 3]. In an independent research, Artur Ekert proposed a different approach to establish a secret key between two parties based on the correlations of two entangled particles [4].

\section{Quantum key distribution}

A cryptographic channel comprises of two distant parties that share a communication channel so that they can communicate securely and privately despite the presence of an eavesdropper. The two parties are typically labeled Alice and Bob, two fictitious characters first popularized by the same Rivest, Shamir, and Adleman [5] who propounds the RSA scheme. The search for an unbreakable cipher remained the "Holy Grail" of cryptography until 1949 when it was shown that one could achieve secure communication theoretically via the one-time-pad (OTP) method (Vernam, 1926) as long as the two users, Alice and Bob, share a sufficiently long random string that is kept secret from Eve. Note that for the OTP scheme to be theoretically secured, it is important that the random string used as the one-time pad is as long as the message itself, and that it is used once and never reused [6].

\section{Springer}

(c) The Author(s). 2021 Open Access This article is licensed under a Creative Commons Attribution 4.0 International License, which permits use, sharing, adaptation, distribution and reproduction in any medium or format, as long as you give appropriate credit to the original author(s) and the source, provide a link to the Creative Commons licence, and indicate if changes were made. The images or other third party material in this article are included in the article's Creative Commons licence, unless indicated otherwise in a credit line to the material. If material is not included in the article's Creative Commons licence and your intended use is not permitted by statutory regulation or exceeds the permitted use, you will need to obtain permission directly from the copyright holder. To view a copy of this licence, visit http://creativecommons.org/licenses/by/4.0/. 
The protocol of distributing such a long key in a onetime pad in the presence of an eavesdropper, typically called Eve, is known as the key distribution problem. Traditionally, such one-time pad are carefully duplicated and distributed to two distant parties through various means, like through a courier, for instance. However, this distribution method suffers from various espionage tactics and it is never very secure. Moreover, once an eavesdropper has access to the one-time pad, he or she can easily copy the contents without either Alice or Bob knowing.

In this review, we survey the landscape for chip-based quantum key distribution. We focus primarily on our two recent work in the area: one on continuous variable $\mathrm{QKD}$ and another on measurement device independent (MDI)QKD.

\section{Chip-based technologies}

The spectacular success of microelectronics has shown that there is an enormous potential for turning basic physics into applications through miniaturization. Silicon photonic technology offers many unparalleled benefits including small size, low cost, low power consumption, and well-established batch fabrication techniques [7]. Indium phosphide (InP) [8], lithium niobate $\left(\mathrm{LiNbO}_{3}\right)$ [9] and potassium titanyl phosphate (KTP) [10] have been used to fabricate on-chip lasers and fast modulators. Silicon technology provides low-loss delay lines and fiber-chip couplers. However, the technology lacks rapid modulation [11, 12]. Silicon relies on well-established microfabrication techniques which is ideally suited for on-chip photonic components [13-15].

\section{$4 \quad$ BB84}

The theory behind BB84 is well documented [16-20]. Alice randomly transmit random bits based on one of the two bases: $\{|H\rangle,|V\rangle\}$ or $\{|+\rangle,|-\rangle\}$ corresponding to bits $\{|0\rangle,|1\rangle\}$ respectively. When Bob receives the photon, he randomly selects one of the two bases to measure. Naturally, if he selects the correct basis, he gets the correct transmitted bit. However, if he does not selects the correct basis, he incurs an error. Alice and Bob then reveal their bases but not their transmitted bits and discard all bits in which Alice and Bob uses different bases. On average, half of the bits transmitted are lost. To ensure further safety in the transmission, they then chooses to verify their bits (by revealing their bit publicly) and immediately discard those bits. This is the process of reconciliation. If there is no eavesdropper, they should always agree on these bits. Any deviation from perfect fit then gives them an idea on the amount of eavesdropping.

The BB84 protocol has been demonstrated experimentally with linear optics over long distances through optical fibers and free space. The first experiment is done with
Alice controlling two pockel cells so that she is able to transmit the four polarization states [17]. Since then, there have been many experimental confirmation of QKD over longer and longer distances through fibers [21, 22], free space [23, 24], and satellites [25-27].

In Ref. [8], the researchers implement the BB84 QKD protocol with time-bin encoding on a chip. With this scheme, a photon encodes the state $|0\rangle$ in the first time-bin and a second photon encodes the state $|1\rangle$ in the second time-bin. The state $|+\rangle$ denotes the superposition of the first and second time-bin photon with zero relative phase. The state $|-\rangle$ denotes the superposition of being in the first and second time-bin with a relative phase of $\pi$. The BB84 protocol transmits one of two orthogonal states chosen at random and encoded in one of two randomly chosen non-orthogonal bases: the Z-basis $\{|0\rangle,|1\rangle\}$ and the X-basis $\{|+\rangle,|-\rangle\}$.

In Ref. [8], the BB84 QKD protocol is implemented with time-bin encoding on a chip. In this scheme, the state $|0\rangle$ is encoded by a photon in the first time-bin and the state $|1\rangle$ is encoded by a photon in the second time-bin. The state $|+\rangle$ is encoded as a superposition of the first and second time-bin photon with zero relative phase. The state $|-\rangle$ is encoded as superposition of being in the first and second time-bin with a relative phase of $\pi$. The BB84 protocol transmits one of two orthogonal states chosen at random, encoded in one of two randomly chosen non-orthogonal bases: the Z-basis $\{|0\rangle,|1\rangle\}$ and the X-basis $\{|+\rangle,|-\rangle\}$.

In the experiment in Ref. [8], the transmitter chip (using InP) consists of an on-chip tunable laser, formed from two distributed Bragg reflectors (DBR) and a semiconductor optical amplifier (SOA). The continuous wave singlemode laser source has a coherence time $>1.5$ ns with a side-mode suppression ratio of $>50 \mathrm{~dB}$ It operates at a wavelength of $1550 \mathrm{~nm}$ with a $10 \mathrm{~nm}$ tuning range. Short electrical pulses are applied to the reverse biased EOPM in the first Mach-Zehnder interferometer (MZI) and optical pulse with $<150$ ps duration and $30 \mathrm{~dB}$ extinction ratio are generated.

The receiver chip comprises alternating layers of $\mathrm{SiO}_{2}$ and $\mathrm{Si}_{3} \mathrm{~N}_{4}$ and etched onto a substrate to create a waveguide structure for guiding light with a high index-contrast at a low loss $(0.5 \mathrm{~dB} / \mathrm{cm})$. The coupling loss between chip and fiber is also low at $2 \mathrm{~dB}$, providing a total loss $9 \mathrm{~dB}$ for BB84 configuration.

In Ref. [8], they actually implemented three different protocols: BB84, coherent one-way (COW) [28] and differential phase shift (DPS) [29]. In all cases, the performances are comparable to the state-of-the-art current fiber and bulk optical systems that have been achieved with a key rates of $345 \mathrm{kbps}$ (BB84), $311 \mathrm{kbps}$ (COW) [8] and $565 \mathrm{kbps}$ (DPS). Similar experiments have also been demonstrated elsewhere [30-33]. 


\section{MDI-QKD}

\subsection{Theory}

Measurement-device-independent quantum key distribution (MDI-QKD) employs an untrusted relay to prevent the communication channel from side-channel attacks commonly encountered in earlier QKD protocols.

To understand MDI-QKD, we follow the nice description in Ref. [34]. To do this, we first introduce an EinsteinPodolsky-Rosen (EPR)-based QKD protocol. Alice and Bob first prepares an EPR pair and sends half of it to an untrusted third party, Charles. Charles then performs an entanglement swapping operation [35, 36] on the incoming signals via a Bell state measurement (BSM), and then broadcast his measurement results. On completion of the measurement, Alice and Bob measure their halves of the EPR pairs with two conjugate bases (the rectilinear basis $Z$, or the diagonal basis $X$ ) that they select at random. By doing so they can determine whether or not Charles is honest. For this, they can compare a randomly chosen subset of their data to test if it satisfies the expected correlations associated with the Bell state declared by Charles.

As shown in the excellent review, Ref. [37], this protocol can also be implemented in a "time-reversal" fashion. This is so because Charles' operations commute with those of Alice and Bob. Therefore, one can reverse the order of the measurements. That is, it is not necessary that Alice and Bob wait for Charles's results in order to measure their halves of the EPR pairs, but they can measure them beforehand. Note that Charles' BSM is only used to check the parity of Alice's and Bob's bits and, therefore, it does not reveal any information about the individual bit values. This rephrases the original EPR-based QKD protocol into an equivalent prepare-and-measure scheme where Alice and Bob directly send Charles BB84 states and Charles performs the measurements. Most importantly, like in the original EPR-based QKD protocol, Alice and Bob can test the honesty of Charles by just comparing a random portion of their signals.

\subsection{Experiment}

Experimental demonstration of MDI-QKD has been performed in Refs. [38, 39] with bulk optics over long distances. The set-ups are ideal for a quantum network. In Ref. [38], a time-bin phase-encoding MDI-QKD scheme is realized, whereas in Ref. [39], the researchers assess the feasibility of MDI-QKD using the decoy-state protocol proposed by Wang [40].

Recently, we have realized a fully chip-based MDI-QKD system (see Fig. 1). We were not the only group to do it on photonic chip. There are also two other excellent pieces of work: the first in China led by Feihu $\mathrm{Xu}$ and Jianwei Pan [41] and the second in Bristol led by Mark Thompson, John Rarity, and Chris Erven [42]. Here, we describe our system. Our system comprises two transmitter chips (Alice and Bob) and one receiver chip (Charlie). The key components of the transmitter chip are the intensity modulators for the decoy states, phase modulators, and polarization modulators. These devices are fully integrated into a single chip. The receiver chip integrates polarization-independent beam splitters (BS) and polarizing beam splitters (PBS). In our experiment, we demonstrate a key rate per pulse of $2.923 \times 10^{-6}$ which is sufficient for low-error quantum communications.

For the transmitter chip, a pulse-modulated 1542.3815$\mathrm{nm}$ frequency-locked laser is coupled into the silicon waveguide through a grating coupler. The first MachZehnder interferometer (MZI) modulator modifies the laser intensity to create signal and decoy states, and another phase modulator implements random phase modulations of the input pulses. Polarization states $|H\rangle,|V\rangle,|+\rangle$ and $|-\rangle$ are encoded by a polarization modulator consisting of an MZI modulator, a path-topolarization convertor (PPC), and phase shifters at the PPC input arms. Light is coupled out of the chip through an adiabatic tapered waveguide coupler and a lensed fiber with a $3-\mu \mathrm{m}$ spot diameter. The edge coupler is designed with a cross-section of $200 \mathrm{~nm} \times 220 \mathrm{~nm}$ to minimize the polarization-dependent loss (PDL). After attenuation, the weak coherent pulses from the two transmitter chips reach the receiver chip (Charlie) via optical fiber spools. The receiver chip consists of a polarizationindependent BS and two PBS. Before entering the receiver chip, the pulses are compensated for polarization drift by polarization controllers in order to maximize the HongOu-Mandel (HOM) interference between the transmitted photons from Alice and Bob chips. Finally, Bell states are measured with single-photon detectors, and all coincidence events for secure key generation are publicly announced. The security of MDI-QKD is guaranteed because the measured outcomes do not contain any information on the secret key encoding.

Both the MDI-QKD transmitter and receiver chips were designed and fabricated by using advanced silicon photonic fabrication techniques, which utilized silicon-oninsulator (SOI) wafer with a top silicon layer of $220 \mathrm{~nm}$ and a buried oxide layer (BOX) of $3 \mu \mathrm{m}$. The top silicon was etched to form grating coupler. Slab layer of ridge waveguide in PPC and other components were fabricated using inductively coupled plasma-reactive ion etching (ICP-RIE). Subsequently, strip waveguide was formed by etching through the remaining silicon. On completion of the waveguide etching, silicon oxide was deposited on the silicon chip. Titanium nitride (TiN) is then added to form the waveguide heaters. Aluminum ( $\mathrm{Al}$ ) was then deposited to provide the necessary electrical connection between external power source and waveguide heaters. Finally, we etch isolation trenches in order to prevent 


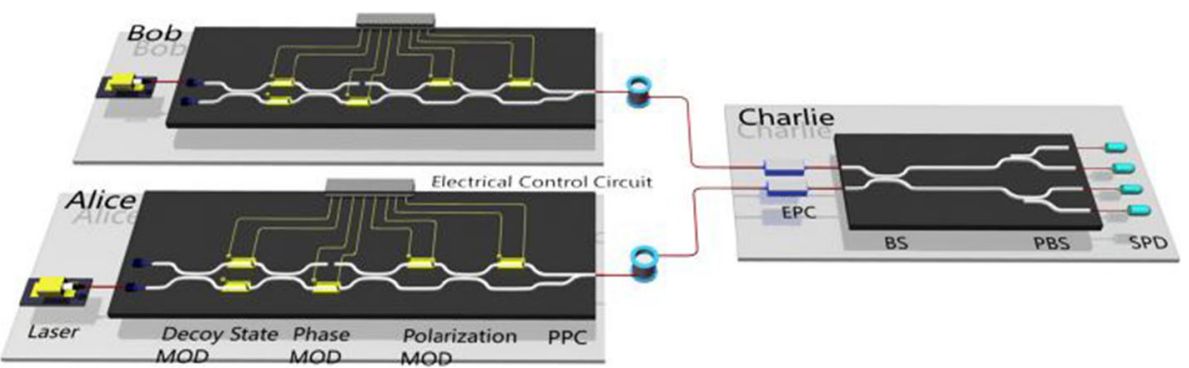

Fig. 1 Schematic diagram of the silicon photonic chip-based MDI QKD system. The system comprises three silicon photonic chips (Alice, Bob, and Charlie) among which Alice and Bob chips are the transmitters and Charlie chip is the server. Alice and Bob chips consist of intensity modulators, phase modulators, and polarization modulators, which are capable of generating phase-randomized signal and decoy-state weak coherent pulses in BB84 polarization states. Charlie's chip is made up of a polarization-independent beam splitter and two polarizing beam splitters and it is capable of performing Bell state measurement of the incoming states with off-chip single-photon detectors (SPDs). MOD, modulator; PPC,

path-to-polarization converter; EPC, electrically driven polarization controller

thermal crosstalk between adjacent heaters. The intensity modulators, phase modulators, and polarization modulators were integrated onto a single transmitter chip. Figure $2 \mathrm{a}$ and $\mathrm{b}$ illustrate the optical micrographs of the transmitter and receiver chip, respectively. Figure 2c shows the transmitter chip packaged on a printed circuit board (PCB).

The MDI-QKD architecture is naturally suited for multi-user QKD networks [43], since the most expensive and intricate component-the measurement device-can be placed in an untrusted relay and shared among many QKD users. Therefore, MDI-QKD has been widely recognized as a promising quantum communication technology for star-type secured networks $[34,44]$.

\section{CV-QKD}

Continuous variable QKD or CV QKD refers to quantum communication with weak coherent pulses and homodyne detections. Compared with discrete variable QKD, continuous variable QKD appears to be more suitable for photonic chip integration due to its compatibility with existing telecom technologies. The advantages and disadvantages of discrete QKD and CV QKD is welldocumented $[45,46]$. Yet, the security analysis of this technique is a lot more complicating compared to discrete-variable QKD despite the simplicity in the actual protocol.

There are two prepare-and-measure continuous variable QKD or Gaussian QKD: (i) an entanglement based scheme using two-mode squeezed states and (ii) a scheme based on coherent states and heterodyne detections.

In the entanglement-based scheme, Alice prepares a two-mode squeezed vacuum state. Alice prepares a coherent state, whose displacement vector is Gaussian distributed in $x$ and $p$, by applying a heterodyne measurement at her share of the two-mode squeezed state.

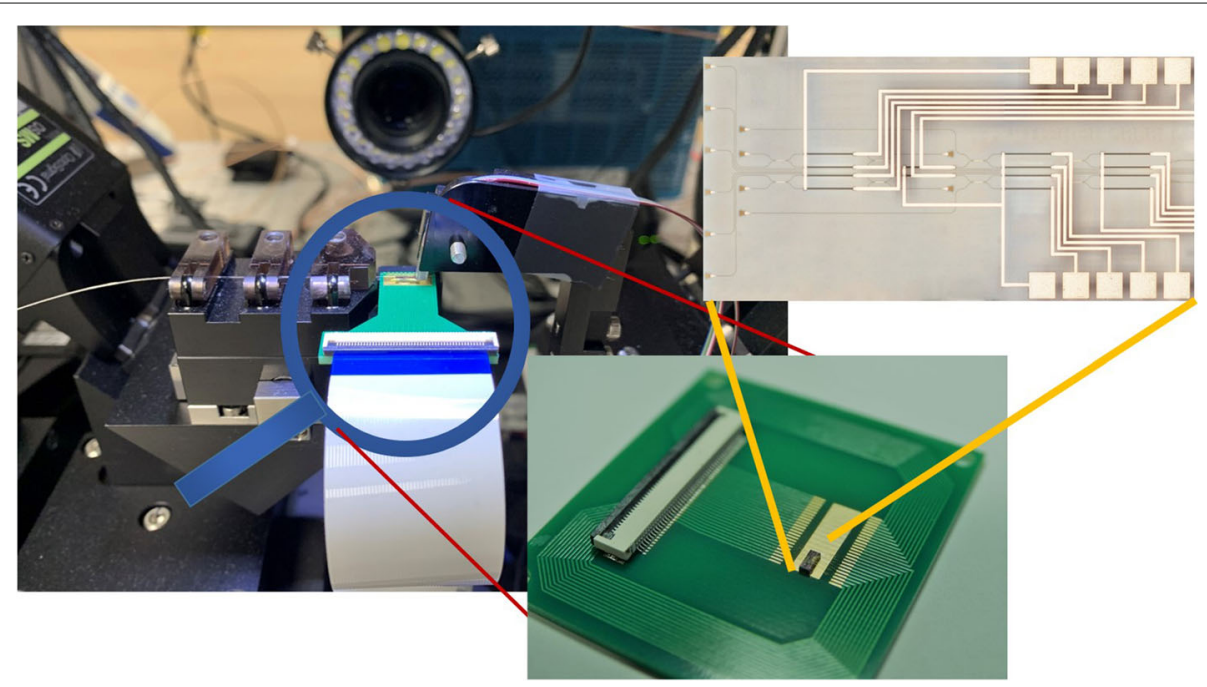

Fig. 2 Picture showing the size of the MDI-QKD chip with integrated photonic circuits together with the peripheral set up. Insets shows the zoom-in version of the tiny chip 
Bob then applies a homodyne measurement on mode B, measuring quadrature $x$ or $p$.

Experimental CV QKD has already been performed over long distances of 80 to $100 \mathrm{~km}[47,48]$ with bulk optics. However, less experimental efforts in chip-based technologies have been performed. Given that CV QKD is fully compatible with existing fiber communications, the advent of chip-based technologies for CV QKD will be a defining step towards a better integration with existing network.

In Ref. [9], we provided a proof-of-principle chip-based CV-QKD system that is capable of producing a secret key rate of $0.14 \mathrm{kbps}$. The protocol is sufficiently robust against collective attack over a simulated distance of 100 $\mathrm{km}$ in fiber. This demonstration offers new possibilities for scalable low-cost portable quantum networks.

Figure 3 shows a schematic diagram of the silicon photonic CV-QKD chip. In the protocol, the transmitter chip (often labeled as Alice) is comprised of a 1550-nm continuous-wave laser coupled to a waveguide with a grating coupler. To control the input laser intensity, we introduce a modulator that serves as an attenuator. We then split the input laser into two separate paths using a 1:99 directional coupler: a weak and a strong beam. The weaker one acts as signal and the stronger one serves as the local oscillator (LO). The signal path is then modulated with an amplitude modulator (AM) and a phase modulator $(\mathrm{PM})$ to generate a series of coherent state $\left|x_{A}+i p_{A}\right\rangle$, where $x_{A}$ and $p_{A}$ are Gaussian distributed random numbers. A digital filter and demodulator then extracts the information from one of the sideband frequencies. As in Ref. $[49,50]$, information encoding is done with a modulation of the continuous light signal on the sideband ranging from 1-10 MHz. Moreover, in order to keep the relative phase between the signal path and the local oscillator (LO) path after transmission, the modulated signal and LO are multiplexed into two orthogonal polarization states with a two-dimensional grating coupler. After the receiver (Bob) gets the signal transmitted over a line with a transmittance $T$, he then compensates the polarization drift with a polarization controller followed by a demultiplexing of the signal and the LO with another two-dimensional grating coupler. Finally, Bob arbitrarily selects to measures $x$ or $p$ quadrature with the on-chip homodyne detector and filters out the required frequency.

The Heisenberg uncertainty principle provides a fundamental basis for security of CV-QKD between the $x$ and $p$ quadratures due to the fact that the quadratures do not commute. Therefore, any attempt by the eavesdropper's (Eve) obtain information by to measuring one quadrature results in noise in the other quadrature. In this way, the amount of information of the key leaked to Eve is bound by the noise level detected by Alice and Bob if Eve do not wish to be detected. Clearly, it does not pay for any eavesdropper to be detected.

In our experiment, the homodyne detection efficiency is $\eta=0.498$. We also measure 5 -dB loss of Bob's chip due to an additional $68.3 \%$ drop in efficiency. The total excess noise is $\epsilon=0.0934$ shot-noise units (SNU) at a modulation variance of $V_{\text {mod }}=7.07 \mathrm{SNU}$ and $T=1$. Detector electrical noise is $v_{e l}=0.0691 \mathrm{SNU}$. Symbol rate is $S R=$ $0.8 \mathrm{Mbps}$. These data allow us to estimate the key rate of the current CV-QKD system. We found that, at $90 \mathrm{~km}$, the Shannon raw key rate and Holevo raw key rate are both roughly $10^{3}$. Figure 4 shows the reconciliation efficiency and signal to noise ratio (SNR) values. The secure fraction and the calculated SNR is plotted as a function of transmission distance. These values are comparable to existing benchmarks.

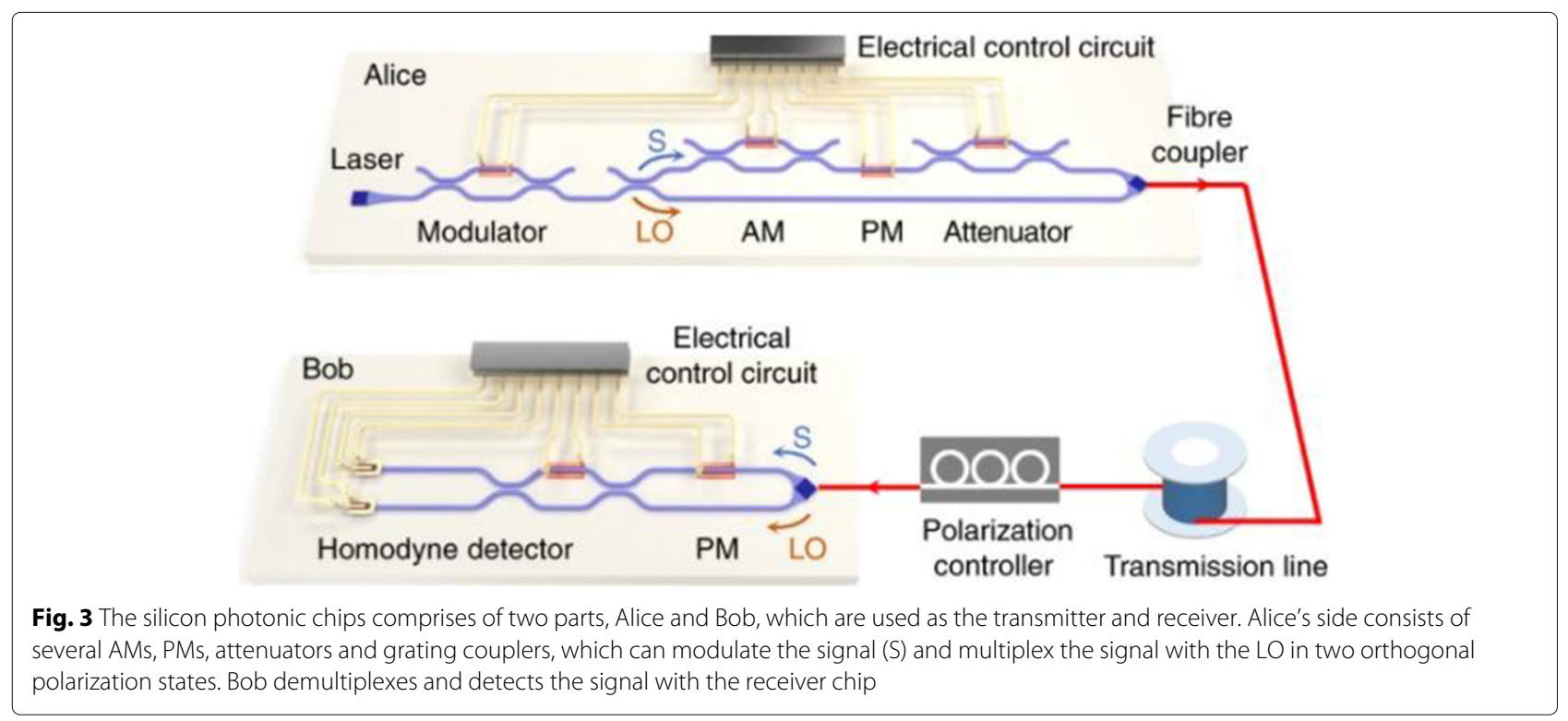



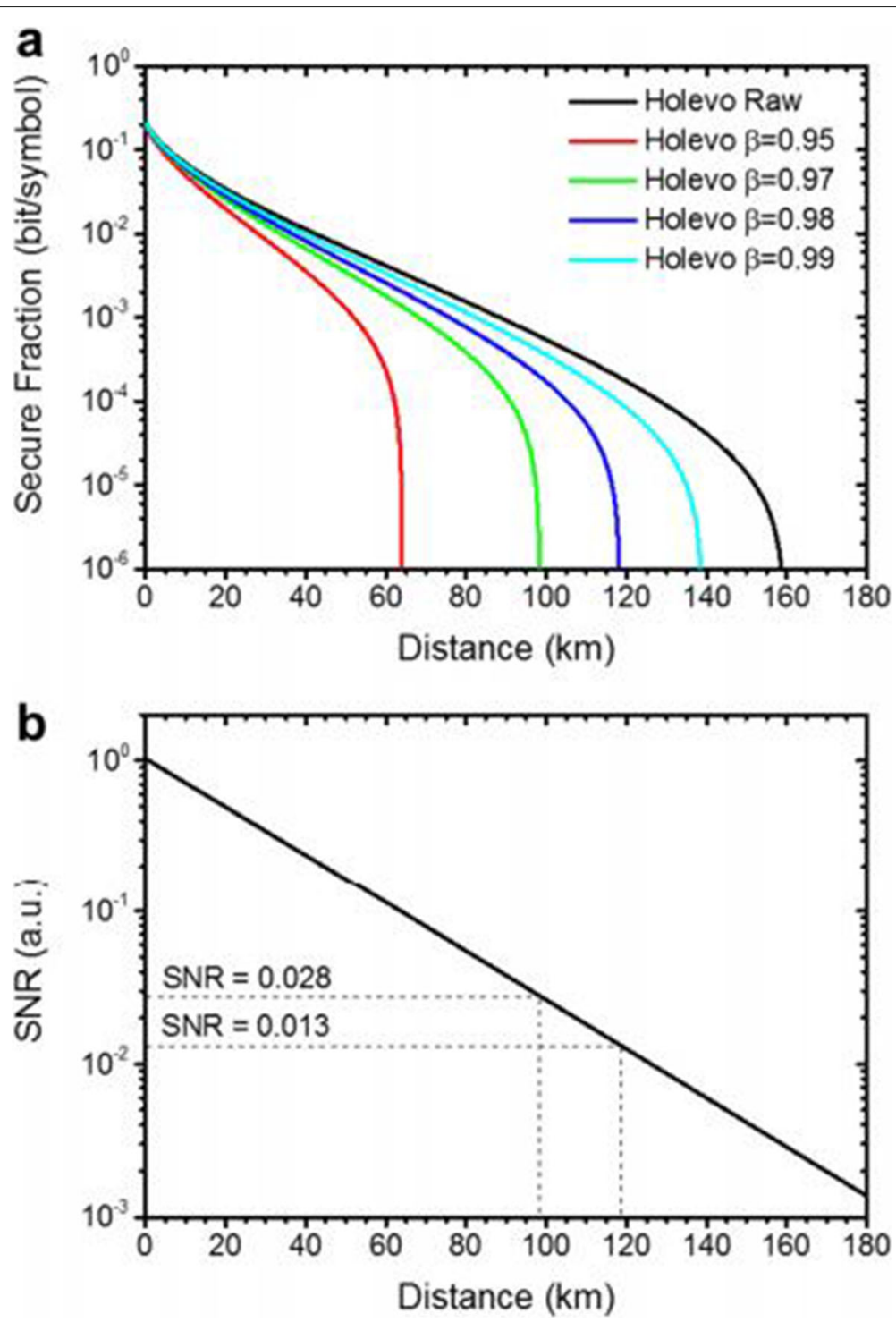

Fig. 4 Reconciliation efficiency and SNR considerations. a The secure fraction as a function of transmission distance. $\mathbf{b}$ The calculated SNR at different simulated fiber transmission distance

\section{Overall prospect and conclusion}

Integrated photonics continues to offer one of the most stable, compact, and robust platforms to miniaturize massive photonic circuits [51]. The mature fabrication processes of silicon chips and its full compatibility with electronic circuits are also advantages for a compact device. This compactness and compatibility with classical devices would also allow QKD devices to be embedded into classical devices without easy detection. Overall, chip-based technologies offer advantages in size compactness, low energy consumption, and a potential for low cost $[7,45]$.

With no electro-optical nonlinearity, many silicon photonic chips currently utilize slow thermo-optical phase modulators for high fidelity state preparation [52]. Yet, high-speed modulation of quantum states in standard silicon photonics appears possible in the near future with carrier- injection and carrier-depletion modulators even though the latter currently does not quite work well in quantum applications. These limitations can however be overcome [52].

Finally, the compatibility of integrated photonic chips with current integrated photonic telecommunication hardware provides a seamless integration with classical communications channels and transceivers. This integration will pave the way for hybrid classical and quantum communications devices [52]. 


\section{Acknowledgment}

This work was supported by the Singapore Ministry of Education (MOE) Tier 3 grant (MOE2017-T3-1-001), the Singapore National Research Foundation (NRF) National Natural Science Foundation of China (NSFC) joint grant (NRF2017NRF-NSFC002-014), and the Singapore National Research Foundation under the Competitive Research Program (NRF-CRP13-2014-01).

\section{Authors' contributions}

All authors contributed equally to the review. The authors read and approved the final manuscript.

\section{Authors' information}

Not applicable

\section{Funding}

See the "Acknowledgements" section: this work is supported by the Singapore Ministry of Education (MOE) Tier 3 grant (MOE2017-T3-1-001), the Singapore National Research Foundation (NRF) National Natural Science Foundation of China (NSFC) joint grant (NRF2017NRF-NSFC002-014), and the Singapore National Research Foundation under the Competitive Research Program (NRF-CRP13-2014-01).

\section{Declarations}

\section{Competing interests}

The authors declare that they have no conflict of interest.

\section{Author details}

${ }^{1}$ Centre for Quantum Technologies, National University of Singapore, Singapore, Singapore. ${ }^{2}$ MajuLab, CNRS-UNS-NUS-NTU International Joint Research Unit, Singapore UMI 3654, Singapore, Singapore. ${ }^{3}$ National Institute of Education, Nanyang Technological University, Singapore 637616, Singapore. ${ }^{4}$ Quantum Science and Engineering Centre (QSec), Nanyang Technological University, Singapore, Singapore. Institute of Microelectronics, Peking University, Beijing, People's Republic of China. ${ }^{6}$ School of Electrical and Electronic Engineering, Nanyang Technological University, Singapore, Singapore. ${ }^{7}$ School of Optoelectronic Science and Engineering, University of Electronic Science and Technology of China, Chengdu 610054, People's Republic of China. ${ }^{8}$ School of Physics and Astronomy, Sun Yat-Sen University, Zhuhai 519082, People's Republic of China. ${ }^{9}$ State Key Laboratory of Low Dimensional Quantum Physics, Department of Physics, Tsinghua University, Beijing 100084, People's Republic of China. ${ }^{10}$ Synergetic Innovation Center of Quantum Information and Quantum Physics, University of Science and Technology of China Hefei, Anhui 230026, People's Republic of China. ${ }^{11}$ Jinan Institute of Quantum technology, SAICT, Jinan 250101, People's Republic of China.

\section{Received: 17 February 2021 Accepted: 26 May 2021}

Published online: 14 June 2021

\section{References}

1. S. Wiesner, Conjugate coding. ACM Sigact News. 15(1), 78-88 (1983)

2. C. H. Bennett, G. Brassard, Proceedings of the IEEE International Conference on Computers, Systems and Signal Processing. (IEEE, New York, 1984)

3. G. Brassard, in IEEE Information Theory Workshop on Theory and Practice in Information-Theoretic Security, 2005. Brief history of quantum cryptography: a personal perspective (IEEE, New York, 2005), pp. 19-23

4. A. K. Ekert, Quantum cryptography based on Bell's theorem. Phys. Rev. Lett. 67(6), 661 (1991)

5. R. L. Rivest, A. Shamir, L. Adleman, A method for obtaining digital signatures and public-key cryptosystems. Commun. ACM. 26(1), 96-99 (1983)

6. C. E. Shannon, Communication theory of secrecy systems. Bell Syst. Tech. J. 28(4), 656-715 (1949)

7. A. Orieux, E. Diamanti, Recent advances on integrated quantum communications. J. Opt. 18(8), 083002 (2016)

8. P. Sibson, C. Erven, M. Godfrey, S. Miki, T. Yamashita, M. Fujiwara, M. Sasaki, H. Terai, M. G. Tanner, C. M. Natarajan, et al, Chip-based quantum key distribution. Nat. Commun. 8(1), 1-6 (2017)
9. G. Zhang, J. Y. Haw, H. Cai, F. Xu, S. Assad, J. F. Fitzsimons, X. Zhou, Y. Zhang, S. Yu, J. Wu, et al, An integrated silicon photonic chip platform for continuous-variable quantum key distribution. Nat. Photonics. 13(12), 839-842 (2019)

10. S. Tanzilli, A. Martin, F. Kaiser, M. P. De Micheli, O. Alibart, D. B. Ostrowsky, On the genesis and evolution of integrated quantum optics. Laser Photonics Rev. 6(1), 115-143 (2012)

11. A. Politi, M. J. Cryan, J. G. Rarity, S. Yu, J. L. O'brien, Silica-on-silicon waveguide quantum circuits. Science. 320(5876), 646-649 (2008)

12. K. M. Davis, K. Miura, N. Sugimoto, K. Hirao, Writing waveguides in glass with a femtosecond laser. Optics letters. 21(21), 1729-1731 (1996)

13. J. G. Huang, H. Cai, Y. Gu, L. K. Chin, J. H. Wu, T. N. Chen, Z. C. Yang, Y. L. Hao, A. Q. Liu, Torsional frequency mixing and sensing in optomechanical resonators. Appl. Phys. Lett. 111(11), 111102 (2017)

14. Y. Z. Shi, S. Xiong, Y. Zhang, L. K. Chin, Y.-Y. Chen, J. B. Zhang, T. Zhang, W. Ser, A. Larrson, S. Lim, et al, Sculpting nanoparticle dynamics for single-bacteria-level screening and direct binding-efficiency measurement. Nat. Commun. 9(1), 1-11 (2018)

15. Y. Shi, S. Xiong, L. K. Chin, J. Zhang, W. Ser, J. Wu, T. Chen, Z. Yang, Y. Hao, B. Liedberg, et al, Nanometer-precision linear sorting with synchronized optofluidic dual barriers. Sci. Adv. 4(1), 0773 (2018)

16. N. Gisin, G. Ribordy, W. Tittel, H. Zbinden, Quantum cryptography. Rev. Mod. Phys. 74(1), 145 (2002)

17. C. H. Bennett, F. Bessette, G. Brassard, L. Salvail, J. Smolin, Experimental quantum cryptography. J. Cryptol. 5(1), 3-28 (1992)

18. R. J. Hughes, D. M. Alde, P. Dyer, G. G. Luther, G. L. Morgan, M. Schauer, Quantum cryptography. Contemp. Phys. 36(3), 149-163 (1995)

19. C. H. Bennett, G. Brassard, A. K. Ekert, Quantum cryptography. Sci. Am. 267(4), 50-57 (1992)

20. P. W. Shor, J. Preskill, Simple proof of security of the BB84 quantum key distribution protocol. Phys. Rev. Lett. 85(2), 441 (2000)

21. D. Rosenberg, J. W. Harrington, P. R. Rice, P. A. Hiskett, C. G. Peterson, R. J. Hughes, A. E. Lita, S. W. Nam, J. E. Nordholt, Long-distance decoy-state quantum key distribution in optical fiber. Phys. Rev. Lett. 98(1), 010503 (2007)

22. C.-Z. Peng, J. Zhang, D. Yang, W.-B. Gao, H.-X. Ma, H. Yin, H.-P. Zeng, T. Yang, X.-B. Wang, J.-W. Pan, Experimental long-distance decoy-state quantum key distribution based on polarization encoding. Phys. Rev. Lett. 98(1), 010505 (2007)

23. J. Wabnig, D. Bitauld, H. Li, A. Laing, J. O'brien, A. Niskanen, Demonstration of free-space reference frame independent quantum key distribution. New J. Phys. 15(7), 073001 (2013)

24. T. Schmitt-Manderbach, H. Weier, M. Fürst, R. Ursin, F. Tiefenbacher, T. Scheidl, J. Perdigues, Z. Sodnik, C. Kurtsiefer, J. G. Rarity, et al, Experimental demonstration of free-space decoy-state quantum key distribution over 144 km. Phys. Rev. Lett. 98(1), 010504 (2007)

25. S.-K. Liao, W.-Q. Cai, W.-Y. Liu, L. Zhang, Y. Li, J.-G. Ren, J. Yin, Q. Shen, Y. Cao, Z.-P. Li, et al, Satellite-to-ground quantum key distribution. Nature. 549(7670), 43-47 (2017)

26. G. Vallone, D. Bacco, D. Dequal, S. Gaiarin, V. Luceri, G. Bianco, P. Villoresi, Experimental satellite quantum communications. Phys. Rev. Lett. 115(4), 040502 (2015)

27. R. Bedington, J. M. Arrazola, A. Ling, Progress in satellite quantum key distribution. NPJ Quat. Inf. 3(1), 1-13 (2017)

28. D. Stucki, N. Brunner, N. Gisin, V. Scarani, H. Zbinden, Fast and simple one-way quantum key distribution. Appl. Phys. Lett. 87(19), 194108 (2005)

29. T. Honjo, H. Takesue, K. Inoue, Differential-phase quantum key distribution experiment using a series of quantum entangled photon pairs. Opt. Lett. 32(9), 1165-1167 (2007)

30. D. Bunandar, A. Lentine, C. Lee, H. Cai, C. M. Long, N. Boynton, N. Martinez, C. DeRose, C. Chen, M. Grein, et al, Metropolitan quantum key distribution with silicon photonics. Phys. Rev. X. 8(2), 021009 (2018)

31. C. Ma, W. D. Sacher, Z. Tang, J. C. Mikkelsen, Y. Yang, F. Xu, T. Thiessen, H.-K. Lo, J. K. Poon, Silicon photonic transmitter for polarization-encoded quantum key distribution. Optica. 3(11), 1274-1278 (2016)

32. J.W. Silverstone, J. Wang, D. Bonneau, P. Sibson, R. Santagati, C. Erven, J. O'Brien, M. Thompson, in 2016 International Conference on Optical MEMS and Nanophotonics (OMN). Silicon quantum photonics (IEEE, New York, 2016), pp. 1-2

33. T. K. Paraïso, I. De Marco, T. Roger, D. G. Marangon, J. F. Dynes, M. Lucamarini, Z. Yuan, A. J. Shields, A modulator-free quantum key distribution transmitter chip. NPJ Quant. Inf. 5(1), 1-6 (2019) 
34. F. Xu, H. Xu, H.-K. Lo, Protocol choice and parameter optimization in decoy-state measurement-device-independent quantum key distribution. Phys. Rev. A. 89(5), 052333 (2014)

35. J.-W. Pan, D. Bouwmeester, H. Weinfurter, A. Zeilinger, Experimental entanglement swapping: entangling photons that never interacted. Phys. Rev. Lett. 80(18), 3891 (1998)

36. M. Zukowski, A. Zeilinger, M. A. Horne, A. K. Ekert, "Event-ready-detectors" Bell experiment via entanglement swapping. Phys. Rev. Lett. 71(26), 4287 (1993)

37. F. Xu, M. Curty, B. Qi, H.-K. Lo, Measurement-device-independent quantum cryptography. IEEE J. Sel. Top. Quant. Electron. 21(3), 148-158 (2014)

38. Y. Liu, T.-Y. Chen, L.-J. Wang, H. Liang, G.-L. Shentu, J. Wang, K. Cui, H.-L. Yin, N.-L. Liu, L. Li, et al, Experimental measurement-device-independent quantum key distribution. Phys. Rev. Lett. 111(13), 130502 (2013)

39. A. Rubenok, J. A. Slater, P. Chan, I. Lucio-Martinez, W. Tittel, Real-world two-photon interference and proof-of-principle quantum key distribution immune to detector attacks. Phys. Rev. Lett. 111(13), 130501 (2013)

40. X.-B. Wang, Three-intensity decoy-state method for device-independent quantum key distribution with basis-dependent errors. Phys. Rev. A. 87(1), 012320 (2013)

41. K. Wei, W. Li, H. Tan, Y. Li, H. Min, W.-J. Zhang, H. Li, L. You, Z. Wang, X. Jiang, et al, High-speed measurement-device-independent quantum key distribution with integrated silicon photonics. Phys. Rev. X. 10(3), 031030 (2020)

42. H. Semenenko, P. Sibson, A. Hart, M. G. Thompson, J. G. Rarity, C. Erven, Chip-based measurement-device-independent quantum key distribution. Optica. 7(3), 238-242 (2020)

43. L. Kong, Z. Li, C. Li, L. Cao, Z. Xing, J. Cao, Y. Wang, X. Cai, X. Zhou, Photonic integrated quantum key distribution receiver for multiple users. Opt. Express. 28(12), 18449-18455 (2020)

44. B. Fröhlich, J. F. Dynes, M. Lucamarini, A. W. Sharpe, Z. Yuan, A. J. Shields, A quantum access network. Nature. 501(7465), 69-72 (2013)

45. F. Xu, X. Ma, Q. Zhang, H.-K. Lo, J.-W. Pan, Secure quantum key distribution with realistic devices. Rev. Mod. Phys. 92(2), 025002 (2020)

46. V. Scarani, H. Bechmann-Pasquinucci, N. J. Cerf, M. Dušek, N. Lütkenhaus, M. Peev, The security of practical quantum key distribution. Rev. Mol. Biotechnol. 81(3), 1301 (2009)

47. P. Jouguet, S. Kunz-Jacques, A. Leverrier, Long-distance continuous-variable quantum key distribution with a gaussian modulation. Phys. Rev. A. 84(6), 062317 (2011)

48. P. Jouguet, S. Kunz-Jacques, A. Leverrier, P. Grangier, E. Diamanti, Experimental demonstration of long-distance continuous-variable quantum key distribution. Nat. Photonics. 7(5), 378-381 (2013)

49. A. M. Lance, T. Symul, V. Sharma, C. Weedbrook, T. C. Ralph, P. K. Lam, No-switching quantum key distribution using broadband modulated coherent light. Phys. Rev. Lett. 95(18), 180503 (2005)

50. Y. Shen, H. Zou, L. Tian, P. Chen, J. Yuan, Experimental study on discretely modulated continuous-variable quantum key distribution. Phys. Rev. A. 82(2), 022317 (2010)

51. J. Wang, F. Sciarrino, A. Laing, M. G. Thompson, Integrated photonic quantum technologies. Nat. Photonics. 14(5), 273-284 (2020)

52. P. Sibson, J. E. Kennard, S. Stanisic, C. Erven, J. L. O'Brien, M. G. Thompson, Integrated silicon photonics for high-speed quantum key distribution. Optica. 4(2), 172-177 (2017)

\section{Publisher's Note}

Springer Nature remains neutral with regard to jurisdictional claims in published maps and institutional affiliations. 